\title{
ON STRONGLY PRIME SUBMODULES
}

\author{
ABDULRASOOL AZIZI
}

Received 13 May, 2015

\begin{abstract}
Let $R$ be a commutative ring with identity and $M$ an $R$-module. A proper submodule $N$ of $M$ is called strongly prime [resp. strongly semiprime], if $((N+R x): M) y \subseteq N$ [resp. $((N+R x): M) x \subseteq N$ ] for $x, y \in M$ implies that $x \in N$ or $y \in N$ [resp. $x \in N$ ]. Strongly prime and strongly semiprime submodules are studied, in this paper.
\end{abstract}

2010 Mathematics Subject Classification: 13A10; 13C99; 13E05; 13F05; 13F15

Keywords: I-maximal submodule, prime submodule, strongly prime submodule, strongly semiprime submodule

\section{INTRODUCTION}

Throughout this paper all rings are commutative with identity and all modules are unitary. Also we consider $R$ to be a ring and $M$ a unitary $R$-module. If $N$ is a submodule of $M$, then $(N: M)=\{t \in R \mid t M \subseteq N\}$.

Recall from [12] that a proper submodule $N$ of $M$ is said to be strongly prime [resp. strongly semiprime], if $((N+R x): M) y \subseteq N$ [resp. $((N+R x): M) x \subseteq N$ ] for $x, y \in M$ implies that $x \in N$ or $y \in N$ [resp. $x \in N$ ].

If $N$ is a strongly prime [resp. strongly semiprime] submodule of $M$ and ( $N$ : $M)=I$, then we say that $N$ is an $I$-strongly prime [resp. $I$-strongly semiprime] submodule of $M$.

A proper submodule $N$ of $M$ is prime if the condition $r a \in N, r \in R$ and $a \in M$ implies that either $a \in N$ or $r M \subseteq N$. In this case, if $P=(N: M)$, we say that $N$ is a $P$-prime submodule of $M$, and it is easy to see that $P$ is a prime ideal of $R$ (see, for example, $[2-8,10,11,13])$.

In [11], another notion of strongly prime submodules was introduced. It is easy to see that for modules over commutative rings with identity, the strongly prime submodules introduced in [11] coincide with the prime submodules. However, the strongly prime submodules in this paper are special cases of prime submodules (see (1)(2)).

In [12, Proposition 1.1], it is shown that:

(1) Every strongly prime submodule is strongly semiprime and also prime.

(c) 2018 Miskolc University Press 
(2) Every maximal submodule is strongly prime.

A characterization of strongly prime submodules is given in (1), and there we prove that the converse of (1) is also correct, see also (2). The converse of (2) is studied in (3), (1), and (6).

Prime submodules have been the subject of numerous publications in the past. So the relations between prime submodules and strongly prime submodules will be helpful in connecting the previous papers on the prime notion for modules to this new notion of prime. Especially there are a few papers studying the form of the elements of the intersection of prime submodules (for example, $[3,7,10,13])$. This is a motivation for studying the equality of the intersection of prime submodules with the intersection of strongly prime submodules in this paper (see (7) and (3)).

The Generalized Principal Ideal Theorem (GPIT) states that if $R$ is a Noetherian ring and $P$ is a minimal prime ideal over an ideal $I$ generated by $n$ elements of $R$, then $h t P \leq n$. The module version of GPIT related to prime submodules has been studied in [6] and slightly in [5]. In [12, Theorem 2.3], it is proved that the module version of GPIT related to strongly prime submodules is true for every Noetherian flat module. This shows that the behavior of height of strongly prime submodules is closer to prime ideals in comparing with prime submodules, and strongly prime submodules behave better than prime submodules for studying the dimension theory in modules.

In [12, Theorem 1.7], it is claimed that any intersection of strongly prime submodules is a strongly semiprime submodule. We show that this claim is incorrect (see (1) and (4)).

The existence of strongly prime submodules are studied in (1), (4), (3), (9). The results (2) and (5) are devoted to studying the existence of strongly semiprime submodules. In (3) and (3) we show that strongly prime submodules exist rarely in injective modules, although (9) shows that they are found frequently in projective modules. We apply the notion of strongly prime submodules and show that if there exists a non-zero module over a primary ring that is both projective and injective, then the ring has only one prime ideal (see (6)).

\section{STRONGLY PRIME AND STRONGLY SEMIPRIME SUBMODULES}

Let $I$ be an ideal of $R$. A submodule $N$ of $M$ is called $I$-maximal, if $(N: M)=I$ and the submodule $N$ is maximal with respect to this property, that is, if $L$ is a submodule of $M$ containing $N$ with $(L: M)=I$, then $L=N$ (see [2] and [10, p. 1810]).

One can easily see that if $N$ is $I$-maximal and $I$ is a prime [resp. maximal] ideal, then $N$ is a prime [resp. maximal] submodule (see [10, Lemma 3.2]).

The following result is a generalization of [12, Proposition 1.1]. This result also shows that strongly prime submodules are exactly $I$-maximal submodules, where $I$ is a prime ideal of $R$. 
Proposition 1. Let $N$ be a submodule of $M$. Then the following are equivalent:

(1) $N$ is a strongly prime submodule of $M$.

(2) $N$ is a strongly semiprime submodule and also a prime submodule of $M$.

(3) $N$ is a strongly semiprime submodule of $M$ and $(N: M)$ is a prime ideal.

(4) $N$ is $(N: M)$-maximal and $(N: M)$ is a prime ideal of $R$.

Proof. ((a) $\Longrightarrow(b))$. See [12, Proposition 1.1].

$((\mathrm{b}) \Longrightarrow(\mathrm{c}))$. Note that for every prime submodule $N$ of $M$, the ideal $(N: M)$ is prime.

$((\mathrm{c}) \Longrightarrow(\mathrm{d}))$. To show that $N$ is $(N: M)$-maximal, suppose that $L$ is a submodule of $M$ containing $N$ with $(L: M)=(N: M)$. Let $x$ be an arbitrary element of $L$. As $N \subseteq(N+R x) \subseteq L$, we have $(N: M) \subseteq((N+R x): M) \subseteq(L: M)=(N: M)$, that is, $((N+R x): M)=(N: M)$. Then $((N+R x): M) x=(N: M) x \subseteq N$, and since $N$ is a strongly semiprime submodule, $x \in N$. This shows that $L=N$, whih completes the proof.

$((\mathrm{d}) \Longrightarrow(\mathrm{a}))$. Let $P=(N: M)$. Since $N$ is $P$-maximal and $P$ is a prime ideal, by [10, Lemma 3.2], $N$ is a prime submodule; so if $((N+R x): M) y \subseteq N$, for $x \in M$ and $y \in M \backslash N$, then $((N+R x): M) \subseteq(N: M)$ and evidently $(N: M) \subseteq$ $((N+R x): M)$. Hence $((N+R x): M)=(N: M)=P$ and since $N$ is $P$-maximal, $x \in(N+R x)=N$, and so $x \in N$. 1.

Example 1. Let $P$ be a maximal ideal of $R$ and $F$ a free $R$ module with $\operatorname{rank} F>$

(1) $F$ contains a $P$-strongly prime submodule.

(2) $F$ contains a $P$-prime submodule that is not strongly prime.

Proof. Let $F=\oplus_{i \in \alpha} R$, and assume that $i_{0} \in \alpha$.

(1) We show that $N=P \oplus\left(\oplus_{i \in \alpha, i \neq i_{0}} R\right)$ is a $P$-strongly prime submodule of $F$. Obviously $P F \subseteq \oplus_{i \in \alpha} P \subseteq N$, that is $P \subseteq(N: F)$, and as $P$ is a maximal ideal of $R$, we have $(N: F)=P$. Now let $L$ be a submodule of $F$ containing $N$ with $(L: F)=$ $(N: F)$. Since $(L: F)=P$ is a maximal ideal, $L$ is a $P$-prime submodule of $F$. If $N \neq L$, then let $\left\{x_{i}\right\}_{i \in \alpha} \in L \backslash N$, and so $x_{i_{0}} \notin P$. Obviously $\left\{\left(1-\delta_{i_{0} i}\right) x_{i}\right\}_{i \in \alpha} \in N \subseteq$ $L$, and thus $x_{i_{0}}\left\{\delta_{i_{0} i}\right\}_{i \in \alpha}=\left\{x_{i}\right\}_{i \in \alpha}-\left\{\left(1-\delta_{i_{0} i}\right) x_{i}\right\}_{i \in \alpha} \in L$, and since $L$ is a $P$-prime submodule of $M$, we have $e_{i_{0}}=\left\{\delta_{i_{0}}\right\}_{i \in \alpha} \in L$. Also evidently for each $j \in \alpha, j \neq i_{0}$, we have $e_{j}=\left\{\delta_{j i}\right\}_{i \in \alpha} \in N \subseteq L$. Therefore $L=F$, which is a contradiction. This shows that $N$ is a $P$-strongly prime submodule submodule of $F$.

(2) By $[4$, Corollary 2.9(i)] if $M$ is a flat module and $P M \neq M$, then $P M$ is a $P$-prime submodule of $M$. So the submodule $P F$ is a $P$-prime submodule of $F$ and note that $P F \subset N$ and $(N: F)=P$, hence $P F$ is not a strongly prime submodule of $F$.

Recall that an ideal $I$ of $R$ is semiprime if $I=\sqrt{I}$. Strongly semiprime submodules of a module are characterized in the following. 
Proposition 2. A submodule $N$ of $M$ is strongly semiprime if and only if $(N: M)$ is a semiprime ideal, and $N$ is $(N: M)$-maximal.

Proof. $(\Longrightarrow)$. To show that $(N: M)$ is a semiprime ideal, on the contrary, let $r \in \sqrt{(N: M)} \backslash(N: M)$. Suppose that $1<n$ is the smallest positive integer with $r^{n} \in(N: M)$, and let $z \in r^{n-1} M \backslash N$. Then $R z \subseteq r^{n-1} M \subseteq r M$, and so $((N+R z)$ : $M) \subseteq((N+r M): M)$. Hence:

$$
\begin{aligned}
& ((N+R z): M) z \subseteq((N+r M): M) r^{n-1} M \\
= & r^{n-1}((N+r M): M) M \subseteq r^{n-1}(N+r M) \subseteq N,
\end{aligned}
$$

and as $N$ is a strongly semiprime submodule, $z \in N$, which is impossible.

To show that $N$ is $(N: M)$-maximal, suppose that $L$ is a submodule of $M$ containing $N$ with $(L: M)=(N: M)$. Let $x$ be an arbitrary element of $L$. Then $((N+R x): M) \subseteq((L+R x): M)=(L: M)=(N: M)$. Then $((N+R x): M) x \subseteq$ $(N: M) x \subseteq N$, an so $x \in N$, because $N$ is a strongly semiprime submodule. This shows that $L=N$, and thus $N$ is $(N: M)$-maximal.

$(\Longleftarrow)$ Suppose $((N+R x): M) x \subseteq N$, where $x \in M$. Let $r_{1}, r_{2} \in((N+R x): M)$. Then $r_{1} r_{2} M \subseteq r_{1}(N+R x) \subseteq N+R r_{1} x \subseteq N+((N+R x): M) x \subseteq N$, and so $r_{1} r_{2} \in(N: M)$. Consequently $((N+R x): M)^{2} \subseteq(N: M)$, and so $((N+R x)$ : $M) \subseteq \sqrt{(N: M)}=(N: M) \subseteq((N+R x): M)$, which implies that $N+R x=N$, by our assumption. Thus $x \in N$.

The following corollary shows that if $\left\{N_{i}\right\}_{i \in \alpha}$ is a family of distinct $P$-strongly prime submodules of $M$ with $|\alpha|>1$, then $\cap_{i \in \alpha} N_{i}$ is never a strongly semiprime submodule. This corollary rejects [12, Theorem 1.7], which claims that any intersection of strongly prime submodules is a strongly semiprime submodule.

Corollary 1. Let $\left\{N_{i}\right\}_{i \in \alpha}$ be a family of $P$-strongly prime submodules of $M$. Then $\cap_{i \in \alpha} N_{i}$ is a strongly semiprime submodule if and only if $N_{i}=N_{j}$ for all $i, j \in \alpha$.

Proof. $(\Longrightarrow)$. Let $B=\cap_{i \in \alpha} N_{i}$. Note that $(B: M)=\cap_{i \in \alpha}\left(N_{i}: M\right)=P=\left(N_{j}\right.$ : $M$ ) for each $j \in \alpha$. Since $B$ is strongly semiprime, by (2) it is ( $B: M)$-maximal and $B \subseteq N_{j}$ with $(B: M)=\left(N_{j}: M\right)$, so $N_{j}=B$ for all $j \in \alpha$, which completes the proof.

$(\Longleftarrow)$. Is evident.

Corollary 2. For a ring $R$, the following are equivalent:

(1) Spec $(R)$ is a chain.

(2) Every strongly semiprime submodule of any $R$-module $M$ is strongly prime.

Proof. $((1) \Longrightarrow(2))$. Every two prime ideals of $R$ are comparable, so $\sqrt{(N: M)}$ is an intersection of a chain of prime ideals, thus it is a prime ideal. By (2), $(N: M)=$ $\sqrt{(N: M)}$, thus it is a prime ideal. Now by (1), $N$ is a strongly prime submodule. 
$((2) \Longrightarrow(1))$. Let $P$ and $Q$ be two prime ideals of $R$. Then $\sqrt{P \cap Q}=\sqrt{P} \cap$ $\sqrt{Q}=P \cap Q$, that is, $P \cap Q$ is a semiprime ideal, and so by our assumption it is a prime ideal. Now if $P \nsubseteq Q$ and $Q \nsubseteq P$, then consider $p \in P \backslash Q$ and $q \in Q \backslash P$. Thus $p q \in P \cap Q$, but $p \notin P \cap Q$, and $q \notin P \cap Q$.

Recall that a commutative ring is primary if its zero ideal is primary (see [1, Chapter 4]). The set of zero-divisors of $R$ is $\mathcal{Z}(R)=\{r \in R \mid \exists 0 \neq s \in R, r s=0\}$. Also $\mathcal{N}(R)=\left\{r \in R \mid \exists n \in \mathbb{N}, r^{n}=0\right\}$.

A characterization of primary rings is given in the following lemma.

Lemma 1. The following are equivalent:

(1) $\mathcal{Z}(R) \subseteq \mathcal{N}(R)$.

(2) $\mathcal{Z}(R)=\mathcal{N}(R)$, and it is a prime ideal.

(3) $R$ is a primary ring.

Proof. ((1) $\Longrightarrow(2))$. We know that $\mathcal{Z}(R)$ is a union of prime ideals, and $\mathcal{N}(R)$ is the intersection of all prime ideals. Let $\mathcal{Z}(R)=\cup_{i \in \alpha} P_{i}$, where each $P_{i}$ is a prime ideal, and let $i_{0} \in \alpha$. Then $\cup_{i \in \alpha} P_{i}=\mathcal{Z}(R) \subseteq \mathcal{N}(R) \subseteq P_{i_{0}} \subseteq \cup_{i \in \alpha} P_{i}$.

$((2) \Longrightarrow(3)$ and $((3) \Longrightarrow(1))$ are obvious.

Proposition 3. Let $R$ be a primary ring, $M$ a divisible $R$-module and $N$ a submodule of $M$. Then the following are equivalent:

(1) $N$ is a maximal submodule.

(2) $N$ is a strongly prime submodule.

(3) $N$ is a strongly semiprime submodule.

(4) $N$ is a maximal submodule and $(N: M)$ is the unique prime ideal of $R$.

Proof. ((1) $\Longrightarrow(2) \Longrightarrow(3)))$ see [12, Proposition 1.1].

$((4) \Longrightarrow(1))$. Is obvious.

((3) $\Longrightarrow(4))$. Let $N$ be a strongly semiprime submodule of $M$ and $L$ a proper submodule of $M$ containing $N$. We show that $(N: M)=(L: M)$.

On the contrary, let $r \in(L: M)) \backslash(N: M)$. By (2), $(N: M)$ is a semiprime ideal, so $\mathcal{Z}(R) \subseteq \mathcal{N}(R) \subseteq \sqrt{(N: M)}=(N: M)$; and hence $r \in R \backslash \mathcal{Z}(R)$. Since $M$ is divisible, $M=r M \subseteq L \subseteq M$, which is a contradiction. Thus $(N: M)=(L: M)$, and by (2), $N$ is ( $N: M)$-maximal, therefore $N=L$, and so $N$ is a maximal submodule of $M$. This also implies that $(N: M)$ is a maximal ideal.

If $t \in(N: M) \backslash \mathcal{Z}(R)$, then as $M$ is divisible, $M=t M \subseteq N$, which is impossible. Hence $(N: M) \subseteq \mathcal{Z}(R) \subseteq \mathcal{N}(R) \subseteq P$ for each prime ideal $P$ of $R$, and as $(N: M)$ is maximal, $(N: M)=P$, the ring $R$ has a unique prime ideal $(N: M)$.

Note 4 . The following corollary shows that in a divisible module over an integral domain (particularly in a vector space), even the intersection of two distinct maximal (strongly prime) submodules is not strongly semiprime, because it is not a maximal submodule. The following result is also a generalization of [12, Proposition 1.3]. 
Corollary 3. Let $R$ be an integral domain and $N$ a submodule of $M$. Then the following are equivalent:

(1) $M$ is an injective module and $N$ is a maximal submodule.

(2) $M$ is a divisible module and $N$ is a strongly prime submodule.

(3) $M$ is a divisible module and $N$ is a strongly semiprime submodule.

(4) $N$ is a maximal submodule and $R$ is a field.

Proof. ((1) $\Longrightarrow(2) \Longrightarrow(3))$ and $((4) \Longrightarrow(1))$ are obvious.

$((3) \Longrightarrow(4))$. The proof is given by (3).

Example 2. Let $\mathbb{R}$ be the field of real numbers and consider $M=\mathbb{R}^{2}$. By (3), the strongly prime submodules of $M$ are exactly the maximal submodules of $M$, and we know that the maximal submodules of $M$ are exactly the lines passing through the origin. Hence although $M$ is a Noetherian module over a Noetherian ring, it has infinitely many minimal strongly prime submodules. Note that according to [11, Theorem 4.2], a Noetherian module over a Noetherian ring has finitely many minimal prime submodules.

For an ideal $I$ of $R$, a primary decomposition $I=\cap_{i=1}^{n} Q_{i}$ will be called coprime, if either $n=1$ or $Q_{i}$ 's are coprime; equivalently when $\sqrt{Q_{i}}$ 's are coprime. When this is the case, because of the Second Uniqueness Theorem (see [1, Theorem 4.10]), $I$ has a unique minimal primary decomposition. For example, in any semilocal ring $R$, for each $n \in \mathbb{N}$, the ideal $(\mathscr{f}(R))^{n}$ has a coprime primary decomposition. Also evidently each ideal of an Atrinian ring or a Noetherian domain of dimension one has a coprime primary decomposition. Let $S=K\left[x_{1}, x_{2}, x_{3}, \cdots\right]$, where $K$ is a field and $x_{1}, x_{2}, x_{3}, \cdots$ are independent indeterminates and let $I=S\left(x_{1}-c_{1}\right)^{n_{1}}\left(x_{1}-\right.$ $\left.c_{2}\right)^{n_{2}}\left(x_{1}-c_{3}\right)^{n_{3}} \cdots\left(x_{1}-c_{m}\right)^{n_{m}}$, where $c_{1}, c_{2}, c_{3}, \cdots, c_{m}$ are distinct element of $K$, and $n_{1}, n_{2}, n_{3}, \cdots, n_{m} \in \mathbb{N}$. Then the ideal $I$ of $S$ has a coprime primary decomposition. Indeed $\cap_{i=1}^{m} S\left(x_{1}-c_{i}\right)^{n_{i}}$ is a coprime primary decomposition of $I$. Thus in the non-Noetherian infinite dimensional ring $R=S / I$, the zero ideal has a coprime primary decomposition.

Theorem 1. Let the zero ideal of $R$ have a coprime primary decomposition. If $M$ is a divisible $R$-module, then every strongly prime submodule of $M$ is maximal.

Proof. If the zero ideal of $R$ is primary, then the proof is given by (3). Now suppose that $0=\cap_{i=1}^{n} Q_{i}$ is a coprime primary decomposition of the zero ideal. Consider the natural ring homomorphism $\varphi: R \rightarrow\left(R / Q_{1}\right) \times\left(R / Q_{2}\right) \times \cdots \times\left(R / Q_{n}\right)$, $\varphi(r)=\left(r+Q_{1}, r+Q_{2}, \cdots, r+Q_{n}\right)$. Since $0=\cap_{i=1}^{n} Q_{i}$ and $Q_{i}$ 's are coprime, by [1, Proposition 1.10], $\varphi$ is a ring isomorphism. Hence without loss of generality, we can assume that $R=R_{1} \times R_{2} \times \cdots \times R_{n}$, where each $R_{i}$ is a primary ring. Put $M_{1}=(1,0, \cdots, 0) M, M_{2}=(0,1,0, \cdots, 0) M, \cdots, M_{n}=(0,0, \cdots, 0,1) M$. One can easily see that $M_{1}$ is an $R_{1}$-module with the multiplication $r_{1}((1,0, \cdots, 0) m)=(1,0, \cdots, 0)\left(\left(r_{1}, 0, \cdots, 0\right) m\right)$ for each $r_{1} \in R_{1}$ and $m \in M$. Similarly each $M_{i}$ is an $R_{i}$-module and $M \cong M_{1} \times M_{2} \times \cdots \times M_{n}$ as $R$-modules. So we 
can assume that $M=M_{1} \times M_{2} \times \cdots \times M_{n}$ is a divisible $R_{1} \times R_{2} \times \cdots \times R_{n}$-module, and each $M_{i}$ is an $R_{i}$-module. Evidently for each $r_{1} \in R_{1}$, we have:

(1) $r_{1} \in \mathcal{Z}\left(R_{1}\right)$ if and only if $\left(r_{1}, 1,1, \cdots, 1\right) \in \mathcal{Z}(R)$.

(2) $r_{1} M_{1}=M_{1}$ if and only if $\left(r_{1}, 1,1, \cdots, 1\right) M=M$.

Therefore as $M$ is a divisible $R$-module, $M_{1}$ is a divisible $R_{1}$-module. Similarly $M_{i}$ is a divisible $R_{i}$-module, for each $i>1$.

Let $T$ be a submodule of $M$. It is easy to see that:

(3) $T=T_{1} \times T_{2} \times \cdots \times T_{n}$, where each $T_{i}$ is a submodule of $M_{i}$ as an $R_{i}$-module.

(4) $(T: M)=\left(T_{1}: M_{1}\right) \times\left(T_{2}: M_{2}\right) \times \cdots \times\left(T_{n}: M_{n}\right)$.

Let $N$ be a strongly prime submodule of $M$. Then by (3), $N=N_{1} \times N_{2} \times \cdots \times N_{n}$, where each $N_{i}$ is a submodule of $M_{i}$ as an $R_{i}$-module. We show that there exists a $1 \leq j \leq n$ such that $N_{j}$ is a strongly prime submodule of $M_{j}$ and $N_{i}=M_{i}$ for each $i \neq j$.

By [9, p. 6, Exercise 1.2], the prime ideals of $R$ are of the form $R_{1} \times R_{2} \times \cdots \times$ $R_{j-1} \times P_{j} \times R_{j+1} \times \cdots \times R_{n}$, where $P_{j}$ is a prime ideal of $R_{j}$. Thus as by (4) and (1), $(N: M)=\left(N_{1}: M_{1}\right) \times\left(N_{2}: M_{2}\right) \times \cdots \times\left(N_{n}: M_{n}\right)$ is a prime ideal of $R$, there exists a $1 \leq j \leq n$ such that $\left(N_{j}: M_{j}\right)$ is a prime ideal of $R_{j}$ and $\left(N_{i}: M_{i}\right)=R_{i}$ for each $i \neq j$. Hence $N_{i}=M_{i}$ for each $i \neq j$. Now we show that $N_{j}$ is a strongly prime submodule of $M_{j}$.

Let $L_{j}$ be a submodule of $M_{j}$ containing $N_{j}$ with $\left(N_{j}: M_{j}\right)=\left(L_{j}: M_{j}\right)$. Consider $L=M_{1} \times M_{2} \times \cdots \times M_{j-1} \times L_{j} \times M_{j+1} \times \cdots \times M_{n}$. By (4), $(N: M)=(L: M)$, and since $N$ is a strongly prime submodule of $M$ and $N \subseteq L$, by (1), we have $N=L$, which implies that $N_{j}=L_{j}$. Thus again by (1), $N_{j}$ is a strongly prime submodule of $M_{j}$. Now as $R_{j}$ is a primary ring, by (3), $N_{j}$ is a maximal submodule of $M_{j}$. Hence by (3), $N=M_{1} \times M_{2} \times \cdots \times M_{j-1} \times N_{j} \times M_{j+1} \times \cdots \times M_{n}$ is a maximal submodule of $M$.

The set of all prime [resp. strongly prime] submodules of an $R$-module $M$ is denoted by $\operatorname{Spec}(M)$ or $\operatorname{Spec}_{R}(M)$ [resp. SSpec$\left.(M) \operatorname{or~SSpec}_{R}(M)\right]$. Evidently if $M$ is finitely generated, then $\operatorname{SSpec}_{R}(M) \neq \varnothing$.

Proposition 5. Let the zero ideal of $R$ have a coprime primary decomposition and $\operatorname{dim} R>0$. Then there does not exist any non-zero finitely generated divisible $R$-module. Particularly there does not exist any non-zero finitely generated injective $R$-module.

Proof. Let $M$ be a non-zero finitely generated divisible $R$-module. According to the proof of (1), we can assume that $R=R_{1} \times R_{2} \times \cdots \times R_{n}$, where each $R_{i}$ is a primary ring, and $M=M_{1} \times M_{2} \times \cdots \times M_{n}$ is an $R_{1} \times R_{2} \times \cdots \times R_{n}$-module, where each $M_{i}$ is a non-zero finitely generated divisible $R_{i}$-module, and $\operatorname{sosSpec}_{R_{i}}\left(M_{i}\right) \neq$ $\varnothing$. Then (3) implies that $R_{i}$ has a unique prime ideal $P_{i}$, and hence the prime ideals of $R$ are exactly $\widehat{P}_{i}=R_{1} \times R_{2} \times \cdots \times R_{i-1} \times P_{i} \times R_{i+1} \times \cdots \times R_{n}$, for $1 \leq i \leq n$. 
Evidently $\widehat{P}_{i} \not \subseteq \widehat{P_{j}}$, for any $i \neq j$. This shows that $\operatorname{dim} R=0$, which is a contradiction.

Let $S=R \backslash \mathcal{Z}(R)$. Then $S$ is a multiplicatively closed subset of $R$, and the ring $R_{S}$ is called the total quotient ring of $R$. Since $S$ contains no zero divisors, the natural map $R \rightarrow R_{S}$ is injective, so $R_{S}$ is a ring extension of $R$, and evidently $R_{S}$ is an $R$-module.

Lemma 2. Let $N$ be a submodule of $M$ with $(N: M)=P$.

(1) $N$ is a maximal submodule if and only if $N$ is a strongly prime submodule and $P$ is a maximal ideal of $R$.

(2) If $P$ is a maximal ideal, then there exists a $P$-strongly prime (maximal) submodule of $M$ containing $N$.

Proof. (1) The proof is evident by (1).

(2) Note that $M / N$ is a vector space over the field $R / P$, so it has a maximal subspace, say $\mathcal{M} / N$. Then $\mathcal{M}$ is a maximal submodule of $M$ containing $N$, and as $P=(N: M) \subseteq(\mathcal{M}: M)$, we have $P=(\mathcal{M}: M)$.

Corollary 4. Let the zero ideal of $R$ have a coprime primary decomposition, and let $K$ be the total quotient ring of $R$. Then the following are equivalent:

(1) $\operatorname{dim} R=0$.

(2) There exists a $P$-strong prime $R$-submodule of $K$, for each minimal prime ideals $P$ of $R$.

(3) $K=R$.

Proof. Evidently $K$ is a divisible $R$-module. Let $0=\cap_{i=1}^{n} Q_{i}$ be a coprime primary decomposition of the zero ideal and $\sqrt{Q_{i}}=P_{i}$. Then the minimal prime ideals of $R$ are exactly $P_{1}, P_{2}, \cdots, P_{n}$, also $\mathcal{Z}(R)=\cup_{i=1}^{n} P_{i}$.

((a) $\Longrightarrow\left(\right.$ b)). As $\operatorname{dim} R=0$, the ideals $P_{1}, P_{2}, \cdots, P_{n}$ are all of the maximal ideals of $R$. Obviously each $P_{i}$ is a proper submodule of $K$ as an $R$-module. We show that $\left(P_{i}: K\right)=P_{i}$, and so by (2)(2), there exists a $P_{i}$-strongly prime $R$-submodule of $K$, which completes the proof.

Let $a / s \in K$, where $a \in R$ and $s \in R \backslash \mathcal{Z}(R)$. As $s \notin \mathcal{Z}(R)=\cup_{i=1}^{n} P_{i}$, it is a unit element of $R$, and hence for each $p_{i} \in P_{i}$, we have $p_{i}(a / s)=\left(s^{-1} p_{i} a\right) / 1 \in P_{i}$. Consequently $P_{i} K \subseteq P_{i}$, that is $P_{i} \subseteq\left(P_{i}: K\right)$, and since $P_{i}$ is a maximal ideal, $P_{i}=\left(P_{i}: K\right)$.

((b) $\Longrightarrow(\mathrm{c}))$. By our assumption there exists a $P_{i}$-strongly prime submodule $N_{i}$ of $K$ as an $R$-module, and (1) implies that $N_{i}$ is a maximal submodule of $K$. Hence for each $1 \leq i \leq n$, the ideal $P_{i}$ is a maximal ideal $R$, and so the ideals $P_{1}, P_{2}, \cdots, P_{n}$, are all of the maximal ideals of $R$. Now let $b / t \in K$, where $b \in R$ and $t \in R \backslash \mathcal{Z}(R)$. As $t \notin \mathcal{Z}(R)=\cup_{i=1}^{n} P_{i}$, it is a unit element of $R$, and hence $b / t=\left(t^{-1} b\right) / 1 \in R$. Consequently $K=R$. 
((c) $\Longrightarrow($ a)). Since $K=R$, the $R$-module $K$ is a non-zero finitely generated divisible module, and so by (5), $\operatorname{dim} R=0$.

Recall from [13] that an $R$-module $M$ is said to be special, if for each maximal ideal $\mathfrak{M}$ of $R$, every $a \in \mathfrak{M}$ and any $m \in M$, there exist $c \in R \backslash \mathfrak{M}$ and $k \in \mathbb{N}$ such that $c a^{k} m=0$. Semi-simple modules (direct sum of simple modules), locally Artinian modules (modules in which every cyclic submodule is Artinian) and semiArtinian modules (modules of which every homomorphic image has a nonzero simple submodule) are special (see [13, Section 3]).

\section{Proposition 6.}

(1) Every strongly prime submodule of a special module is a maximal submodule.

(2) $\operatorname{dim} R=0$ if and only if every strongly prime submodule of any $R$-module is maximal.

Proof. (1) Let $N$ be a strongly prime submodule of $M$ and $\mathfrak{M}$ a maximal ideal of $R$ containing $(N: M)$. Consider $m \in M \backslash N$, and $a \in \mathfrak{M}$. Since $M$ is special, there exist $c \in R \backslash \mathfrak{M}$ and $k \in \mathbb{N}$ such that $c a^{k} m=0$. Then $c a^{k} m \in N$ and as $N$ is a prime submodule and $c \notin(N: M)$ and $m \notin N$, we have $a \in(N: M)$, and so $(N: M)=\mathfrak{M}$. This shows that $(N: M)$ is a maximal ideal of $R$, and so by (2)(1), $N$ is a maximal submodule of $M$.

(2) Let $\operatorname{dim} R=0$. By [13, Theorem 3.5], every module over a zero dimensional ring is special, so the proof is given by part (1).

For the 'if part' it is enough to consider $R$ as an $R$-module.

\section{Example 3.}

(1) Consider the $\mathbb{Z}$-module $M=\mathbb{Z} / n \mathbb{Z}$, where $n$ is a positive integer. Evidently each semiprime ideal of $\mathbb{Z}$ is an ideal generated by a square free integer. It is easy to see that strongly semiprime submodules of $M$ are of the form $k \mathbb{Z} / n \mathbb{Z}$, where $k$ is a square free integer with $k \mid n$.

(2) More generally let $R$ be a Dedekind domain and consider the cyclic $R$ module $M=R / I$, where $I$ is a proper ideal of $R$. If the prime factorization of $I$ is $P_{1}^{\alpha_{1}} P_{2}^{\alpha_{2}} \cdots P_{m}^{\alpha_{m}}$, then the strongly semiprime submodules of $M$ are $P_{1}^{\beta_{1}} P_{2}^{\beta_{2}} \cdots P_{m}^{\beta_{m}} / I$, where each $\beta_{i}$ is either 0 or 1 , and at least for one $1 \leq i \leq m, \beta_{i}=1$.

(3) Each strongly prime submodule of the $\mathbb{Z}$-module $M=\mathbb{Z} / n \mathbb{Z}$ is of the form $p \mathbb{Z} / n \mathbb{Z}$, where $p$ is a prime factor of $n$, so they are exactly the maximal submodules of $M$.

The following result guarantees the existence of an $I$-strongly semiprime submodule in a finitely generated module $M$, for any semiprime ideal $I$ containing Ann $M$.

Theorem 2. Let $B$ be a submodule of a finitely generated R-module M. If ( $B$ : $M) \subseteq I$, where $I$ is a proper semiprime ideal of $R$, then there exists an I-strongly semiprime submodule of $M$ containing $B$. 
Proof. Let $\mathcal{T}=\{C \leq M \mid B \subseteq C,(C: M) \subseteq I\}$. By Zorn's Lemma $\mathcal{T}$ has a maximal element, say $N$. We prove that $N$ is an $I$-strongly semiprime submodule of $M$.

According to [9, Ex. 2.2., p. 13], if $M$ is finitely generated and $I$ an ideal of $R$, then $\sqrt{(I M: M)}=\sqrt{\operatorname{Ann}(M)+I}$. Hence:

$$
\begin{gathered}
((N+I M): M)=\left(I \frac{M}{N}: \frac{M}{N}\right) \subseteq \sqrt{\left(I \frac{M}{N}: \frac{M}{N}\right)}=\sqrt{\operatorname{Ann}\left(\frac{M}{N}\right)+I} \\
=\sqrt{(N: M)+I}=I .
\end{gathered}
$$

So $N+I M \in \mathcal{T}$, and since $N$ is a maximal element of $\mathcal{T}$, we have $N=N+I M$. Hence $I \subseteq((N+I M): M)=(N: M) \subseteq I$, and thus $(N: M)=I$.

Now suppose that $L$ is a submodule of $M$ containing $N$ with $(L: M)=(N: M)$. Then as $N$ is a maximal element of $\mathcal{T}$, evidently $L=N$. Hence by (2), $N$ is a strongly semiprime submodule of $M$.

Corollary 5. Let $B$ be a submodule of a finitely generated $R$-module $M$. Then there exists a $\sqrt{(B: M)}$-strongly semiprime submodule of $M$ containing $B$.

The following result is a generalization of [5, Lemma 4] or [10, Theorem 3.3].

Lemma 3. Let $B$ be a submodule of $M$ and $P$ a prime ideal of $R$. Then $B$ is contained in a $P$-strongly prime submodule, if one of the following holds:

(1) $M$ is a finitely generated $R$-module and $(B: M) \subseteq P$.

(2) $M$ is a special $R$-module and $B$ is a $P$-prime submodule of $M$.

Proof. (1) By (2), there exists a $P$-strongly semiprime submodule $N$ of $M$ containing $B$, and since $(N: M)=P$ is a prime ideal, (1) implies that $N$ is a $P$-strongly prime submodule of $M$.

(2) Follow the proof of (6)(1) to see that if $B$ is a $P$-prime submodule of a special module, then $P$ is a maximal ideal. Now by (2)(2), $B$ is contained in a $P$-strongly prime and containing $B$.

Lemma 4. [2, Theorem 2.8] If $M$ is a finitely generated module, then every $P$ prime submodule of $M$ is an intersection of $P$-maximal submodules of $M$.

Let $B$ be a proper submodule of an $R$-module $M$. The intersection of all prime submodules of $M$ containing $B$ is denoted by $\operatorname{rad}(B)$ or $\operatorname{rad}_{R}(B)$. If $M$ has no prime submodules containing $B$, then we consider $\operatorname{rad}(B)=M$. Also according to [12], the intersection of all strongly prime submodules of $M$ containing $B$ is denoted by s- $\operatorname{rad}(B)$ or s- $\operatorname{rad}_{R}(B)$. If $M$ has no strongly prime submodules containing $B$, then we say s- $\operatorname{rad}(B)=M$.

The following result shows that s- $\operatorname{rad}(B)$ coincides with $\operatorname{rad}(B)$, for finitely generated or special modules. 
Proposition 7. $s$-rad $(B)=\operatorname{rad}(B)$, for every submodule $B$ of $M$, if $M$ is a finitely generated or a special module.

Proof. By (3), $\operatorname{rad}(B)=M$ if and only if s-rad(B)=M. Now suppose that $\operatorname{rad}(B) \neq M$. As each strongly prime submodule is a prime submodule, $\operatorname{rad}(B) \subseteq$ s-rad $(B)$.

First suppose that $M$ is a finitely generated module. For proving the converse inclusion, note that by (4), every $P$-prime submodule of $M$ is an intersection of Pmaximal submodules of $\mathrm{M}$ and by (1), the $\mathrm{P}$-maximal submodules of $\mathrm{M}$ are exactly the $P$-strongly prime submodules of $M$. Hence every $P$-prime submodule of $M$ is an intersection of strongly prime submodules. This shows that s-rad $(B) \subseteq \operatorname{rad}(B)$, when $M$ is finitely generated.

Now assume that $M$ is a special module. From the proof of (6), for any $P$-prime submodule $N$ of $M$, the ideal $P$ is maximal.

It is well-known that in a vector space the zero subspace is an intersection of maximal subspaces, so for the vector space $M / N$ over the field $R / P$, we have $N / N=\cap_{i \in \alpha} \bar{M}_{i}$, where each $\bar{M}_{i}$ is a maximal subspace of $M / N$. Then for each $i \in \alpha, \bar{M}_{i}=N_{i} / N$, where $N_{i}$ is a maximal submodule of $M$ containing $N$. Hence $N=\cap_{i \in \alpha} N_{i}$, that is, every prime submodule of $M$ is an intersection of strongly prime submodules, and this completes the proof.

Example 4. By (3), the $\mathbb{Z}$-module $\mathbb{Q}$ of all rational numbers has no strongly prime submodules, although the zero submodule is a prime submodule. Hence s-rad $(0)=$ $\mathbb{Q}$ and $\operatorname{rad}(0)=0$, that is, s- $\operatorname{rad}(0) \neq \operatorname{rad}(0)$. This example and also the following result show that (7) does not hold in general.

Theorem 3. For a ring $R$, the following are equivalent:

(1) $\operatorname{dim} R=0$.

(2) $s$-rad $(B)=\operatorname{rad}(B)$, for every $R$-module $M$ and any $B \leq M$.

(3) $s$-rad $(0)=\operatorname{rad}(0)$, for every $R$-module $M$.

Proof. ((i) $\Longrightarrow$ (ii)) is given by (7), and ((ii) $\Longrightarrow$ (iii)) is obvious.

((iii) $\Longrightarrow$ (i)). Let $P$ be a prime ideal of $R$. We show that $P$ is a maximal ideal of $R$. First we prove that s-rad $R / P(0)=\operatorname{rad}_{R / P}(0)$, for every $R / P$-module $M^{\prime}$.

Evidently, we can consider $M^{\prime}$ as an $R$-module, by the natural pull back homomorphism $R \rightarrow R / P$, that is, we define $r x=(r+P) x$, for each $r \in R$ and $x \in M^{\prime}$. Let $N^{\prime}$ be a prime submodule of $M^{\prime}$ as an $R / P$-module. It is easy to check that:

(1) $(*) N^{\prime} / N^{\prime}$ is a prime submodule of $M^{\prime} / N^{\prime}$ as an $R$-module.

(2) (**) If for $N^{\prime} \subseteq N \leq M^{\prime}, N / N^{\prime}$ is a strongly prime submodule of $M^{\prime} / N^{\prime}$ as an $R$-module, then $N$ is a strongly prime submodule of $M^{\prime}$ as an $R / P$ module.

By our assumption and $(*), N^{\prime} / N^{\prime}$ is an intersection of strongly semiprime submodules of $M^{\prime} / N^{\prime}$ as an $R$-module. Now by $(* *), N^{\prime}$ is an intersection of strongly 
semiprime submodules of $M^{\prime}$ as an $R / P$-module. Consequently s-rad $R / P(0)=$ $\operatorname{rad}_{R / P}(0)$, for every $R / P$-module module.

Now suppose that $K$ is the quotient field of $R / P$. It is easy to see that for the $R / P$ module $K$, we have $\operatorname{Spec}_{R / P}(K)=\{0\}$, and so s-rad $R / P(0)=\operatorname{rad}_{R / P}(0)=0$ for the $R / P$-module $K$. Thus by (4), $R / P$ is a field.

Let $S$ be a multiplicatively closed subset of $R$. For any $N \leq M_{S}$, we consider $N^{c}=\{x \in M \mid x / 1 \in N\}$.

Lemma 5. [8, Proposition 1] Let $M$ be an $R$-module and $S$ a multiplicatively closed subset of $R$.

(1) If $N$ is a $P$-prime submodule of $M$ with $P \cap S=\varnothing$, then $N_{S}$ is a $P_{S}$-prime submodule of $M_{S}$ as an $R_{S}$-module and $\left(N_{S}\right)^{c}=N$.

(2) If $T$ is a $Q$-prime submodule of $M_{S}$ as an $R_{S}$-module, then $T^{c}$ is a $Q^{c}$ prime submodule of $M,\left(T^{c}\right)_{S}=T$ and $Q^{c} \cap S=\varnothing$.

The following result is a generalization of [12, Theorem 1.5 and Corollary 1.6].

Proposition 8. Let $M$ be an $R$-module, $S$ a multiplicatively closed subset of $R$, and $N$ a $P$-strongly prime submodule of $M$ with $P \cap S=\varnothing$.

(1) $N_{S}$ is a $P_{S}$-strongly prime submodule of $M_{S}$ as an $R_{S}$-module and $\left(N_{S}\right)^{c}=$ $N$.

(2) If $P$ is a maximal element of $\{I \mid I$ is an ideal of $R$ with $I \cap S=\varnothing\}$, then $N_{S}$ is a maximal submodule of $M_{S}$.

(3) If $M$ is a finitely generated module, then $N_{S}$ is a maximal submodule of $M_{S}$ if and only if $P$ is a maximal element of the set $\{I \mid I$ is an ideal of $R$ with $I \cap$ $S=\varnothing\}$.

(4) Let $T$ be a $Q$-strongly prime submodule of $M_{S}$ as an $R_{S}$-module, where $M$ is finitely generated or a special module, or $Q^{c}$ is a maximal ideal of $R$. Then $T^{c}$ is a $Q^{c}$-strongly prime submodule of $M$.

Proof. (i) As $N$ is a strongly prime submodule, it is a prime submodule, and so according to (5)(i), $N_{S} \neq M_{S}$ and $\left(N_{S}\right)^{c}=N$. Then $N_{S}$ is a strongly prime submodule of $M_{S}$, by [12, Theorem 1.5].

(ii) Let $\mathfrak{M}$ be a maximal ideal of $R_{S}$ containing $P_{S}$. Then $P=\left(P_{S}\right)^{c} \subseteq \mathfrak{M}^{c}$ and $\mathfrak{M}^{c} \cap S=\varnothing$. Now since $P$ is a maximal element of the set $\{I \mid I$ is an ideal of $R$ with $I \cap$ $S=\varnothing\}$, we have $P=\mathfrak{M}^{c}$, and so $P_{S}=\left(\mathfrak{M}^{c}\right)_{S}=\mathfrak{M}$. Thus $P_{S}$ is a maximal ideal of $R_{S}$, and by part (i), $N_{S}$ is a $P_{S}$-strongly prime submodule, so by (2)(1), $N_{S}$ is a maximal submodule of $M_{S}$.

(iii) Let $N_{S}$ be a maximal submodule of $M_{S}$, and suppose

$$
\mathcal{A}=\{I \supseteq P \mid I \text { is an ideal of } R \text { with } I \cap S=\varnothing\} .
$$

By Zorn's lemma $\mathcal{A}$ has a maximal element, say $\mathfrak{M}^{\prime}$. By (3), $N$ is contained in an $\mathfrak{M}^{\prime}$-strongly prime submodule $N^{\prime}$ of $M$. Part (i) implies that $N_{S}^{\prime}$ is a strongly prime 
submodule of $M_{S}$ containing $N_{S}$, and since $N_{S}$ is a maximal submodule, $N_{S}=N_{S}^{\prime}$, and so $N=\left(N_{S}\right)^{c}=\left(N_{S}^{\prime}\right)^{c}=N^{\prime}$. Thus $P=(N: M)=\left(N^{\prime}: M\right)=\mathfrak{M}^{\prime}$, which implies that $P$ is a maximal element of $\{I \mid I$ is an ideal of $R$ with $I \cap S=\varnothing\}$.

(iv) Since $T$ is a $Q$-prime submodule, (5),(ii), implies that $T^{c}$ is a $Q^{c}$-prime submodule of $M$.

If $M$ is finitely generated or a special module, then according to (3), $T^{c}$ is contained in a $Q^{c}$-strongly prime submodule $L$ of $M$. Then $T=\left(T^{c}\right)_{S} \subseteq L_{S}$ and by (5)(i), $\left(L_{S}: M_{S}\right)=Q$. According to (1), $T$ is $Q$-maximal, hence $T=L_{S}$ and by (5)(i), $T^{c}=\left(L_{S}\right)^{c}=L$. Then $T^{c}$ is a $Q^{c}$-strongly prime submodule of $M$.

Now let $Q^{c}$ be a maximal ideal of $R$. If $T^{c}$ is contained in a submodule $U$ of $M$ with $\left(T^{c}: M\right)=(U: M)$, then as $Q^{c}=(U: M)$ is a maximal ideal, $U$ is a prime submodule and (5)(i) implies that $\left(U_{S}: M_{S}\right)=Q$. Evidently $T=\left(T^{c}\right)_{S} \subseteq U_{S}$ and $Q=\left(T: M_{S}\right)=\left(U_{S}: M_{S}\right)$, and as $T$ is a strongly prime submodule, we have $T=U_{S}$, which implies that $T^{c}=U$. This shows that $T^{c}$ is a $Q^{c}$-strongly prime submodule of $M$.

The following example shows that the contraction of a strongly prime submodule is not necessarily strongly prime, in general.

Example 5. Let $P$ be the zero ideal of $\mathbb{Z}$. Then $\mathbb{Z}_{P}=\mathbb{Q}$ and $\mathbb{Q}_{P}=\mathbb{Q}$. So $\operatorname{SSpec}_{\mathbb{Z}_{P}}\left(\mathbb{Q}_{P}\right)=\{0\}$, although $0^{c}=0 \notin \operatorname{SSpec}_{\mathbb{Z}}(\mathbb{Q})$, because $\operatorname{SSpec}_{\mathbb{Z}}(\mathbb{Q})=\varnothing$, by (3).

From (3) we can conclude that injective modules over a primary ring with more than one prime ideal or particularly over an integral domain which is not field, have no strongly prime submodules. The following result shows the opposite of this for projective modules, the dual of injective modules.

Proposition 9. Let $M$ be a non-zero a projective module, and $P$ a prime ideal of $R$. Then $M$ has a strongly prime submodule containing $P M$ if and only if $P M \neq M$.

Proof. Let $P M \neq M$. By [4, Corollary 2.9(i)] if $M$ is a flat module and $P M \neq M$, then $P M$ is a $P$-prime submodule of $M$. Now by (5)(i), $(P M)_{P}$ is a $P_{P}$-prime submodule of $M_{P}$. Particularly this shows that $M_{P} \neq 0$.

Now suppose that $\mathfrak{M}$ is a maximal ideal of $R$ containing $P$. By $\left(M_{\mathfrak{M}}\right)_{P_{\mathfrak{M}}} \cong M_{P}$ and $M_{P} \neq 0$, we have $M_{\mathfrak{M}} \neq 0$. We show that $M_{\mathfrak{M}}$ has a maximal submodule. Since $M_{\mathfrak{M}}$ is a non-zero projective module over the local ring $R_{\mathfrak{M}}$, it is a non-zero free $R_{\mathfrak{M}}$-module. Hence we can assume that $M_{\mathfrak{M}}$ is a direct sum of copies of $R_{\mathfrak{M}}$.

If $M_{\mathfrak{M}}=R_{\mathfrak{M}}$, then obviously $M_{\mathfrak{M}}$ has a maximal submodule (ideal in this case). Now let $M_{\mathfrak{M}}=\oplus_{i \in \alpha} R_{\mathfrak{M}}$, where $|\alpha|>1$, and assume that $i_{0} \in \alpha$, Consider $T=$ $\mathfrak{M}_{\mathfrak{M}} \oplus\left(\oplus_{i \in \alpha, i \neq i_{0}} R_{\mathfrak{M}}\right)$. Obviously $\mathfrak{M}_{\mathfrak{M}} M_{\mathfrak{M}} \subseteq \oplus_{i \in \alpha} \mathfrak{M}_{\mathfrak{M}} \subseteq T$, that is $\mathfrak{M}_{\mathfrak{M}} \subseteq(T$ : $\left.M_{\mathfrak{M}}\right)$, and as $\mathfrak{M}_{\mathfrak{M}}$ is a maximal ideal of $R_{\mathfrak{M}}$, we have $\left(T: M_{\mathfrak{M}}\right)=\mathfrak{M}_{\mathfrak{M}}$. Thus by (2)(2), $T$ is contained in an $\mathfrak{M}_{\mathfrak{M}}$-strongly prime submodule $\mathcal{M}$ of $M_{\mathfrak{M}}$. Therefore as $\left(\mathfrak{M}_{\mathfrak{M}}\right)^{c}=\mathfrak{M}$ is a maximal ideal of $R$, by (8)(iv), $\mathcal{M}^{c}$ is an $\mathfrak{M}$-strongly prime submodule of $M$ containing $P M$. 
The 'only if' part is obvious.

Recall that a ring $R$ is semi simple, if and only if all $R$-modules are projective, if and only if all $R$-modules are injective (see [14, Proposition 4.5]).

Corollary 6. Let the zero ideal of $R$ have a coprime primary decomposition. If there exists a non-zero $R$-module that is both projective and injective, then $R$ has finitely many prime ideals and $\operatorname{dim} R=0$.

Proof. Let $M$ be a non-zero $R$-module that is both projective and injective. By the proof of (1), we can assume that $R=R_{1} \times R_{2} \times \cdots \times R_{n}$, where each $R_{i}$ is a primary ring, and $M=M_{1} \times M_{2} \times \cdots \times M_{n}$, where each $M_{i}$ is a non-zero divisible $R_{i}$-module. We show that $M_{1}$ is a projective $R_{1}$-module.

Let $f_{1}: A_{1} \rightarrow B_{1}$ be a surjective $R_{1}$-module homomorphism, and let $g_{1}: M_{1} \rightarrow$ $B_{1}$ be an $R_{1}$-module homomorphism. For each $2 \leq i \leq n$, consider $A_{i}=B_{i}=$ 0 , and let $A=A_{1} \times A_{2} \times \cdots \times A_{n}$ to $B=B_{1} \times B_{2} \times \cdots \times B_{n}$. Define $f: A \rightarrow$ $B, f\left(a_{1}, a_{2}, a_{3}, \cdots, a_{n}\right)=\left(f_{1}\left(a_{1}\right), 0,0, \cdots, 0\right)$ and $g: M \rightarrow B, g\left(a_{1}, a_{2}, \cdots, a_{n}\right)=$ $\left(g_{1}\left(a_{1}\right), 0,0, \cdots, 0\right)$. Obviously $f$ is a surjective $R$-module homomorphism and $g$ is an $R$-module homomorphism.

As $M$ is a projective $R$-module, there exists an $R$-module homomorphism $h$ : $M \rightarrow A$ with $f \circ h=g$. Consider $\pi_{1}: A \rightarrow A_{1}, \pi_{1}\left(a_{1}, a_{2}, \cdots, a_{n}\right)=a_{1}$, and $\ell_{1}$ : $M_{1} \rightarrow M, \ell_{1}\left(a_{1}\right)=\left(a_{1}, 0, \cdots, 0\right)$, and let $h_{1}: M_{1} \rightarrow A_{1}, h_{1}=\pi_{1} \circ h \circ \ell_{1}$. One can easily see that $h_{1}$ is an $R_{1}$-module homomorphism with $f_{1} \circ h_{1}=g_{1}$. This shows that $M_{1}$ is a projective $R_{1}$-modules. Similarly $M_{i}$ is a projective $R_{i}$-modules, for each $2 \leq i \leq n$.

As $M_{i} \neq 0$ for each $1 \leq i \leq n$, we have $M_{P_{i}} \neq 0$, for some prime ideal $P_{i}$ of $R_{i}$, and by the proof of (9), $\operatorname{SSpec}_{R_{i}}\left(M_{i}\right) \neq \varnothing$. Now as each $M_{i}$ is a divisible $R_{i}$ module, by the proof of (5), $\operatorname{dim} R=0$ and each $R_{i}$ has a unique prime ideal, and therefore $R$ has finitely many prime ideals.

\section{REFERENCES}

[1] M. F. Atiyah and I. Macdonald, Introduction to commutative algebra. Student economy edition., student economy edition ed. Boulder: Westview Press, 2016.

[2] A. Azizi, "Intersection of prime submodules and dimension of modules." Acta Math. Sci., Ser. B, Engl. Ed., vol. 25, no. 3, pp. 385-394, 2005.

[3] A. Azizi, "Radical formula and prime submodules." J. Algebra, vol. 307, no. 1, pp. 454-460, 2007, doi: 10.1016/j.jalgebra.2006.07.006.

[4] A. Azizi, "Height of prime and weakly prime submodules." Mediterr. J. Math., vol. 8, no. 2, pp. 257-269, 2011, doi: 10.1007/s00009-010-0068-6.

[5] A. Azizi and H. Sharif, "On prime submodules." Honam Math. J., vol. 21, no. 1, pp. 1-12, 1999.

[6] S. M. George, R. L. McCasland, and P. F. Smith, "A principal ideal theorem analogue for modules over commutative rings." Commun. Algebra, vol. 22, no. 6, pp. 2083-2099, 1994, doi: $10.1080 / 00927879408824957$.

[7] J. Jenkins and P. F. Smith, "On the prime radical of a module over a commutative ring." Commun. Algebra, vol. 20, no. 12, pp. 3593-3602, 1992, doi: 10.1080/00927879208824530. 
[8] C.-P. Lu, "Spectra of modules." Commun. Algebra, vol. 23, no. 10, pp. 3741-3752, 1995, doi: 10.1080/00927879508825430.

[9] H. Matsumura, Commutative ring theory. Transl. from the Japanese by M. Reid. Paperback ed., paperback ed. ed. Cambridge: Cambridge University Press, 1989.

[10] R. McCasland and M. Moore, "Prime submodules." Commun. Algebra, vol. 20, no. 6, pp. 18031817, 1992, doi: 10.1080/00927879208824432.

[11] R. McCasland and P. Smith, "Prime submodules of Noetherian modules." Rocky Mt. J. Math., vol. 23, no. 3, pp. 1041-1062, 1993, doi: 10.1216/rmjm/1181072540.

[12] A. Naghipour, "Strongly prime submodules." Commun. Algebra, vol. 37, no. 7, pp. 2193-2199, 2009, doi: 10.1080/00927870802467239.

[13] D. Pusat-Yilmaz and P. Smith, "Modules which satisfy the radical formula." Acta Math. Hung., vol. 95, no. 1-2, pp. 155-167, 2002, doi: 10.1023/A:1015624503160.

[14] J. J. Rotman, An introduction to homological algebra. 2nd ed., 2nd ed. Berlin: Springer, 2009. doi: $10.1007 / \mathrm{b} 98977$.

Author's address

Abdulrasool Azizi

Shiraz University, Department of Mathematics, College of Sciences, Shiraz 71457-44776, Iran

E-mail address: aazizi@shirazu.ac.ir a-azizi@yahoo.com 